\title{
Las Matronas de la Beneficencia Municipal y su atención a las mujeres pobres de Málaga (1900-1956)
}

\section{As Parteiras de Assistência Municipal e atenção às mulheres pobres de Málaga (1900-1956)}

\section{The Municipal Welfare Midwives and attention to the poor women of Malaga (1900-1956)}

Ángela Torres Díaz ${ }^{1}$, Cristobal Rengel Díaz ${ }^{2}$ Inmaculada Cubillas Rodriguez ${ }^{3}$, Isabel María Morales Gil ${ }^{4}$

${ }^{1}$ Matrona. Hospital Regional Universitario de Málaga.

${ }^{2}$ Matrona. Hospital Universitario Virgen de la Victoria de Málaga.

${ }^{3}$ Matrona. Hospital Regional Universitario de Málaga

${ }^{4}$ Profesora titular Departamento de Enfermería de la Universidad de Málaga.

Cómo citar este artículo en edición digital: Torres Díaz, A., Rengel Díaz, C., Cubillas Rodríguez, I. y Morales Gil, I.Ma . (2016). Las Matronas de la Beneficencia Municipal y su atención a las mujeres pobres de Málaga (1900-1956). Cultura de los Cuidados

(Edición digital), 20(45). Disponible en: http://dx.doi.org/10.14198/cuid.2016.45.07>

Correspondencia: Ángela Torres Díaz. C/ Soleares no 42. 29130-Málaga

Correo electrónico: angelatravel.01@gmail.com

Recibido: 15/06//2015; Aceptado: 10/02/2016

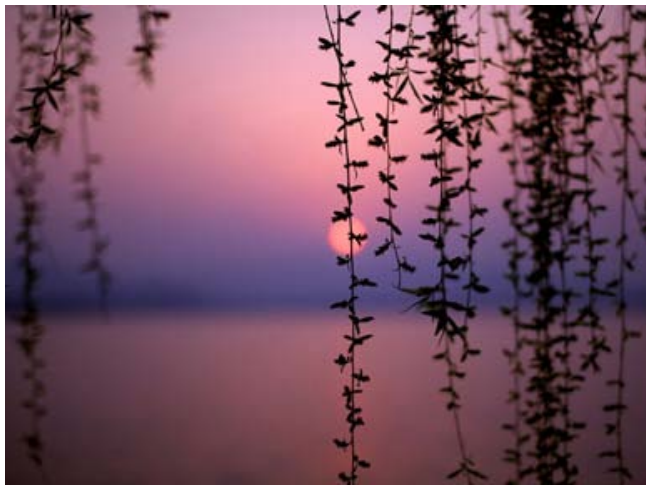

\section{ABSTRACT}

The aim of this study was focused on knowing professional development characteristics and Clinical Practice of Midwives in the $\mathrm{Mu}$ nicipal Charity of Malaga in 1900-1956.

Method and Materials: This was descriptive and historic study. The collection of information has been made through primary and secondary sources.

Results: The process to install the relief houses in the City of Malaga, started in 1862 by Order of the Civil Governor.
Conclusions: The incorporation of the healthcare scenario allowed Midwives coverage during the reproductive process of poor women of Malaga through the Municipal Welfare .

Keywords: history of nursing , charity , midwives.

\section{RESUMO}

O objetivo deste estudo é compreender as características do desenvolvimento profissional e prática clínica das Parteiras Municipal de Assistência Malagueña no período 1900-1956.

Material e Métodos: Foi realizado um estudo descritivo histórico. A coleta de informações foi realizada por meio de fontes primárias e secundárias.

Resultados: O processo para a instalação de casas de socorro na cidade de Malaga, começou em 1862 por ordem do Governador Civil instou o Conselho da Cidade de estabelecer.

Conclusões: A adição do cenário da saúde 
permitiu a cobertura matrona durante o processo reprodutivo de Málaga mulheres pobres através da Previdência Municipal.

Palavras-chave: história da enfermagem , caridade, matronas.

\section{RESUMEN}

El objetivo de este estudio consiste en conocer las características del desarrollo profesional y la práctica clínica de las Matronas de la Beneficencia Municipal Malagueña en el período de 1900-1956.

Material y Método: Se ha realizado un estudio histórico descriptivo. La recogida de información se ha realizado a través de fuentes primarias y secundarias.

Resultados: El proceso para instalar las casas de socorro en la ciudad de Málaga, se inició en 1862 por orden del Gobernador Civil que urgió al Ayuntamiento para que las estableciese.

Conclusiones: La incorporación de la Matrona al escenario sanitario permitió una cobertura durante el proceso reproductivo a las mujeres pobres de Málaga a través de la Beneficencia Municipal.

Palabras clave: historia de la enfermería, beneficiencia, matronas.

\section{INTRODUCCIÓN Y ESTADO DE LA CUESTIÓN}

En 1822, tiene lugar un hecho trascendental para la Historia de la Beneficencia en España, la promulgación de la primera Ley General de Beneficencia, que regularía todo lo referente a esta materia, sirviendo además como base para la futura legislación dictada durante el siglo XIX. Su importancia se debe a que re- presenta el paso definitivo de la Beneficencia a manos de la Administración, tradicionalmente la Beneficencia en España estuvo a cargo de la Iglesia y de particulares, dando a partir de ahora para su ejecución, poder total al Municipio con las Juntas Municipales de Beneficencia para su ejecución (Vidal Galache, 1987). Más tarde en 1849 y la Real Orden de 1864, se determina el establecimiento de la asistencia sanitaria a las mujeres pobres del país, mencionándose en estas Leyes que las parturientas pobres han de ser asistidas en sus casas y que en caso de encontrarse sin recursos y sin hogar debería admitírsela en la casa de socorro municipal o casa de maternidad, dispensándola de todos los cuidados que exigiera su estado y siempre que acreditara su pobreza ante el establecimiento (Martínez Alcubilla, M., 1886). Tras la promulgación de la primera Ley de Beneficencia en cada provincia debería haber una casa de maternidad para refugio de mujeres embarazadas y paridas, se detalla a si mismo en el artículo 47 de la citada Ley que dicha casa de maternidad serviría como escuela de obstetricia para las alumnas que quisieran dedicarse a este arte y que pasado el tiempo que el Gobierno español creyese conveniente no se permitiría ejercer dicho arte a ninguna mujer que no hubiese estudiado en ellas. (Martínez Alcubilla, M., 1886) Respaldando esta Ley a la asistencia al parto por matronas tituladas. Según la estadística de 1858, en la ciudad de Málaga no existía ninguna Casa de parturientas ${ }^{1}$.

Posteriormente y con el comienzo del siglo $\mathrm{XX}$, se promulgaron en España las primeras Leyes de Protección a la Maternidad (Sánchezalbornoz, 1985), hasta ese momento la asistencia al parto era muy precaria y estaba en manos de parteras sin cualificación. La mortalidad materna en 1900 en España por causas atribui-

\footnotetext{
${ }^{1}$ Anuario Nacional de Estadistica(INE). Base Histórica. Siglo XIX.
} 
bles a la maternidad era una de las peores 29 por mil, mientras que en el resto de Europa de 18 por mil (Luque, 2000), las muertes se producían a causa de complicaciones durante la gestación, el parto y sobretodo en el puerperio. Las autoridades sanitarias preocupadas por estas cifras alarmantes emprendieron una política de expansión de una red sanitaria adecuada con profesionales sanitarios cualificados, entre los que la Matrona es la figura central. El Gobierno Español, promulgó El Real DecretoLey del Ministerio de Trabajo y Previsión de 22 de Marzo de 1929 que marcó un antes y un después de la asistencia a las mujeres en su proceso de maternidad. Esta Ley estableció el Seguro de Maternidad que garantizaba entre otras prestaciones, el reconocimiento de la gestante, la asistencia domiciliaria de la Matrona en el momento del parto y el seguimiento de la mujer hasta en los ocho días siguientes al parto, para impedir que por causa de penuria económica, las mujeres fueran asistidas por aficionadas (Vega, Eulalia, 2007).

La atención al nacimiento en los partos normales en la primera mitad del siglo XX, tenía lugar mayoritariamente en los domicilios asistidos por parteras o matronas, que en caso de necesidad debían llamar al médico o trasladar a la mujer a un Hospital. El régimen de asistencia fue el de beneficencia que estaba a cargo de las instituciones municipales (Martínez-Hernáez, Masana, \& DiGiacomo, 2013). A este respecto las mujeres que vivían en áreas rurales tenían muy complicado la asistencia del parto por matronas tituladas, ocurriendo también lo mismo en otras áreas de España (Andina Díaz, 2003) (González Canalejo, 2003) así como el trasladarse a un Hospital cuando surgían dificultades, lo que incidía directamente en un aumento de mortalidad materna y fetal en zonas alejadas de las ciudades. La situación de la mortalidad materna en Málaga era muy similar al resto de España. En el periodo 19141923, se produjeron en la capital un totade 149 muertes de mujeres atribuidas a septicemia puerperal mientras que en la Provincia el total ascendió a 345 muertes atribuibles a la misma causa (Luque, 2000). Así mismo se ha constatado que en el año 1926 en Málaga capital se produjeron un total de 30 muertes maternas atribuibles a causas relacionadas con el embarazo, parto y puerperio; en el área rural el total ascendía a 93 muertes por el mismo motivo, el Gobierno Civil de Málaga, preocupado por la situación emite una carta con fecha de 22 de Mayo de 1928, en la que pone de manifiesto que está dispuesto a combatir el intrusismo profesional, imponiendo multas a las supuestas "parteras ilegales" que venían ejerciendo en

la provincia. Las matronas municipales debían desenvolverse en un mundo laboral construido por profesionales masculinos donde se encontraban con numerosas reticencias y barreras sociales impuestas por el mandato de género y con situaciones de caciquismo y desigualdad.

El objetivo de este estudio consiste en conocer las características del desarrollo profesional y la práctica clínica de las Matronas de la Beneficencia Municipal Malagueña, que prestaron una asistencia a las mujeres pobres de Málaga, en el periodo de 1900-1956.

\section{MATERIAL Y MÉTODO}

Estudio histórico descriptivo. La recogida de información se ha realizado a través de fuentes primarias y secundarias. Entre las fuentes primarias hemos utilizado las siguientes: Estudio de los Reglamentos sobre el personal de la Beneficencia Municipal de Málaga. Boletines Oficiales de la Provincia de Málaga y Boletines Municipales en el periodo de estu- 
dio. En cuanto a las fuentes secundarias: han sido obtenidas de monografías, libros y documentos escritos de Archivos y Bibliotecas consultadas: Archivo Histórico Municipal de Málaga, (AHMM). Archivo Histórico Provincial de Málaga, (AHPM). Archivo Municipal de Vélez-Málaga (AMVM). Archivo Municipal de Antequera (AMA). Archivo Municipal de Ronda (AMR). Archivo Histórico del Colegio de Enfermería de Málaga (ACEM). Biblioteca Nacional, (BN) y Biblioteca de Humanidades de la Universidad de Málaga.

Población de estudio: a través de la documentación de archivo, se identifican y seleccionan Matronas que ejercieron su profesión entre 1900 y 1956 en la Beneficencia Municipal Malagueña.

\section{RESULTADOS}

El proceso para instalar las casas de socoro en la ciudad de Málaga, se inició en 1862 por orden del Gobernador Civil que urgió al Ayuntamiento para que las estableciese. El Reglamento para el Servicio Médico Farmacéutico de la Beneficencia Municipal en Málaga, establecía que las Matronas ingresarían en dicho cuerpo por oposición, guardarían turno de antigüedad, estarían afectas a las Casas de Socorro y bajo las órdenes de sus Directores. Asistirían gratuitamente a domicilio a los partos de las mujeres pobres de su distrito cuando el parto fuese normal, avisando en caso contrario al Médico, el cual atendería a la parturienta u ordenaría su traslado al Hospital ${ }^{2}$. Cuando la mujer era trasladada podía ser atendida en el Hospital Noble, (Beneficencia Municipal) y Hospital Provincial, (Beneficencia de Diputación), previa presentación del papel de inclusión en el padrón de pobre de la ciudad expedido por el Ayuntamiento de la ciudad. Las Matronas de Beneficencia Municipal podían ser Numerarias, asignadas a una plaza y con sueldo, y Supernumerarias sin sueldo, que eran Matronas principiantes que se comprometían a realizar guardias y los servicios de las titulares, sin cobrar nada. Buscaban a cambio darse a conocer y hacerse con una clientela y un prestigio social, no disfrutaban de sueldo en la asistencia pública, pero aumentaban de esta manera su trabajo en la asistencia privada (Giménez Reyna, 1989). La ciudad estaba dividida en tres distritos sanitarios. El número de Casas de Socorro era de cinco en la capital y tres en las barriadas de El Palo, Churriana y Torremolinos. En el momento en que comenzaba el parto en aquellas mujeres de Beneficencia Municipal incluidas en el padrón de pobres, un familiar daba el aviso en la Casa de Socorro correspondiente a su domicilio y el Facultativo del Servicio de Obstetricia del Distrito emitía un boletín para entregar a la Matrona, para que esta fuese avisada del parto y se desplazase el domicilio de la mujer ${ }^{3}$.

La Matrona Municipal llevaba su propio material quirúrgico y utilizaba un equipo o trosseau de partos, que contenía todo lo necesario para la asistencia al parto, este podía ser preparado por las Farmacias o facilitado por el Seguro Obligatorio de Enfermedad. Cuando llegaba la Matrona al domicilio, se preparaba la cama y la habitación donde iba a tener lugar el parto. La cama no podía ser muy blanda y para evitar que la mujer se hundiera, se ponían atravesadas en la cama dos o tres baldas de madera. La cama se recubría con un hule amplio para proteger el colchón (Orengo Díaz del Castillo,

\footnotetext{
${ }^{2}$ Archivo Histórico Municipal de Málaga. Reglamento para el servicio médico y farmaceutico de la Beneficencia municipal. $\operatorname{Lg} 2905$.

${ }^{3}$ Archivo Histórico Municipal de Málaga. Lg 3665
} 
1953). Los partos eran largos y en ocasiones las Matronas debían de permanecer en el domicilio de la mujer durante horas o incluso varios días. Posteriormente al alumbramiento, la Matrona visitaba a la mujer durante el puerperio al menos hasta el $8^{\circ}$ día tras el parto.

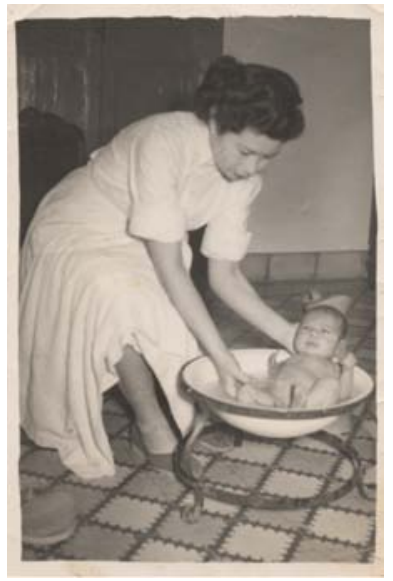

Matrona bañando a un recién nacido (1950)

En el área rural de la provincia de Málaga, el trabajo de las Matronas de Beneficencia era una constante lucha contra las adversidades que rodeaban la asistencia sanitaria en la España de aquellos momentos. La falta de medios, los desplazamientos, solo en casos excepcionales se concedía servicio de carruajes para matronas en casos urgentes y por la noche solamente ${ }^{4}$, unido a la incultura de la población, las dificultades de género y la gran responsabilidad de su trabajo lejos de toda Clínica u Hospital, hacía que el trabajo diario de estas profesionales pioneras del trabajo femenino fuera extremadamente difícil.

Los primeros datos históricos encontrados de la actividad laboral de las Matronas de Beneficencia Municipal de la capital se remontan a finales del siglo XIX, concretamente al año
1895, fue nombrada Matrona de la Beneficencia Municipal, Doña Salvadora Navarro Trujillo, con el haber anual de 1000 pesetas. En 1903, se aumenta a una plaza más la plantilla y se nombra a Doña Rafaela García Díez. Lentamente el número de profesionales en la Beneficencia Municipal se va incrementando en Málaga, en 1914 el número de Matronas era de seis numerarias y tres supernumerarias, hasta un total de ocho Matronas en $1934^{5}$.

A partir de 1947, se vive el lento desmantelamiento de la asistencia por parte de las Matronas de la Beneficencia Municipal de la capital, así consta en el Acta Capitular de 30 de Diciembre de ese año: "No deben ser creadas nuevas plazas de Matronas cuando se tiende a la reducción del Servicio, que necesariamente debe haber disminuido desde la Implantación del Seguro de Maternidad"6.

Los partos realizados por las Matronas de la Beneficencia Municipal Malagueña, se incrementaron rápidamente desde 1926, alcanzando su máximo en 1932, a partir de aquí van descendiendo progresivamente hasta el año 1952.

Gráfico 1: Partos de las Casas de Socorro malagueñas 1918-1952.

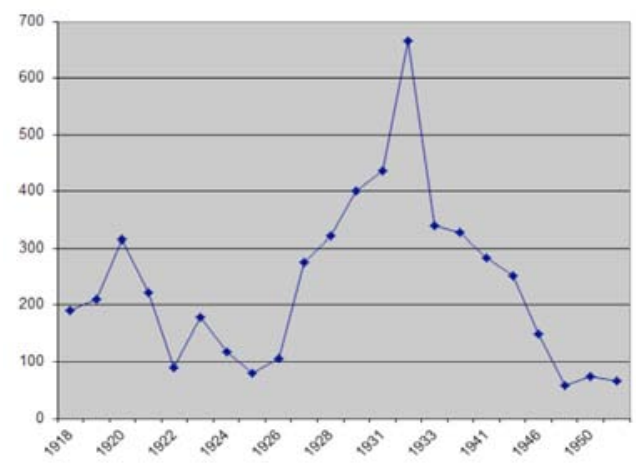

Fuente: Anuario Estadístico Español Base Histórica: Elaboración propia.

\footnotetext{
${ }^{4,5}$ Archivo Histórico Municipal de Málaga. Lg 3654

${ }^{6}$ Archivo Histórico Municipal de Málaga. Acta Capitular 30 de diciembre de 1947
} 
Las primeras oposiciones de Matronas para la Beneficencia Municipal de Málaga de las que se han encontrado datos datan de 1917. En estas oposiciones el temario se componía de cincuenta temas que las profesionales opositoras debían de preparar. El Tribunal para dichas oposiciones estaba formado por el Presidente de la Beneficencia Municipal y tres vocales que eran Médicos de las distintas Casas de Socorro de la ciudad ${ }^{7}$.

No se han encontrado más datos de convocatorias de oposiciones hasta 1940, cuando se convocan tres nuevas plazas de Matronas Supernumerarias sin sueldo. En esta ocasión se designa al Médico Don Cristóbal Porcuna García y a la Matrona Doña María Amador Robledillo, ambos pertenecientes a la Beneficencia Municipal para que formen parte del Tribunal que ha de atender las referidas oposiciones. En estas oposiciones eran condiciones indispensables:

- Ser española.

- Estar en posesión de certificado de buena conducta.

- Aprobar un examen que consistía en tres ejercicios: en el primer ejercicio había que contestar a seis preguntas realizadas al azar sobre el temario, en un mínimo de media hora y un máximo de una hora. En el segundo un ejercicio práctico sobre un maniquí y en el tercero, la resolución de un caso práctico de la especialidad $^{8}$.

Debido al escaso número de Matronas en Málaga capital, las largas distancias que tenían que recorrer, falta de locomoción y la dedicación de 24 horas, se produjeron denuncias a estas profesionales por no haber llegado a tiempo a la asistencia a los partos. Prueba de ello son los distintos documentos históricos encontrados, en la mayoría de los casos por supuesto abandono de su labor asistencial. Encontramos la denuncia a Doña Antonia Segura de Dios, interpuesta por la familia de una parturienta y donde consta: "habiendo quedado sin asistencia la mujer, esta dio a luz un niño muerto". La Matrona dirige un escrito al Sr. Alcalde en la que se defiende de las acusaciones exponiendo: "que en los días 1,2,3 y 4 del mes de Mayo de 1917, se encontraba asistiendo el parto de la mujer de un General de Brigada, muy laborioso y lento". A pesar de estas alegaciones se le suspende con un mes de empleo y sueldo?.

Posteriormente se encuentra la suspensión de la cuarta parte de su sueldo anual a Doña Ángeles Salinas Rodríguez, por no acudir a tiempo a un parto, la noticia tuvo mucha repercusión en el momento y fue publicada el 19 de Noviembre de 1918, en la prensa local ${ }^{10}$.

En otro documento encontramos la denuncia hecha por el Tocólogo Don Francisco Guerrero Andrade hacia la Matrona Doña Encarnación Pérez Rodríguez por haber dejado incumplido el servicio de asistencia a un parto el 8 de Febrero de 1929: "No habiendo aparecido dicha señora tuvo la parturienta que ser asistida por vecinas inexpertas con el escándalo y menoscabo de este Servicio Municipal", Doña Encarnación se defiende diciendo: "que tuvo un accidente en el vuelco de un carruaje produciéndose lesiones que le impedian moverse, siendo asistida por el Médico Sr. Cobos y que encomendó a una compañera para que viera a la parturienta, la cual como el parto iba lento

\footnotetext{
${ }^{7}$ Archivo Histórico Municipal de Málaga. L-23/105

${ }^{8}$ Archivo Histórico Municipal de Málaga. Actas Capitulares .L-348.

${ }^{9}$ Archivo Histórico Municipal de Málaga. Lg 3656.

${ }^{10}$ Archivo Histórico Municipal de Málaga. La Unión Mercantil. 19 de Noviembre de 1918.
} 
encargó a la hermana de la mujer que avisase al Sr. Tocólogo a fin que dispusiese el aviso a la supernumeraria que correspondiese". A pesar de este alegato Doña Encarnación es sancionada con un mes sin sueldo como castigo ${ }^{11}$.

En una carta de esta Matrona dirigida al Colegio Profesional de Matronas en 1929 se queja: "de que el Ayuntamiento de la ciudad solamente tiene para la asistencia a partos a cuatro Matronas en una población de 200.000 habitantes y resulta que hay profesionales que tienen en su distrito cinco mil padrones de pobres.¿Como acudir a todas partes siendo algunas veces requeridas para tres partos de la Beneficencia en un solo día...?, naturalmente que cuando es requerida y no puede prestar la asistencia demandada por encontrarse en otros servicios municipales, claro es que, las parturientas acuciadas por perentoria necesidad del caso $y$ dada su pobreza tienen que acudir a mujeres que por una cantidad ínfima les saca como sea del apuro"12. El Colegio de Matronas solicitó al Ayuntamiento de la ciudad: "sean aumentadas las Matronas municipales en número suficiente para que los servicios puedan prestarse cual corresponde sin que estas pobres que tienen padrón queden sin asistencia"13.
Doña Encarnación fue elegida Presidenta del Colegio el 10 de julio de 1930, convirtiéndose en la segunda Presidenta del Colegio de Matronas. Cesó en el cargo el 30 de Abril de 1932. Fue nombrada nuevamente Presidenta del Colegio en Mayo de 1936 hasta el seis de Octubre de $1949^{14}$.

Las sanciones a Matronas se siguen manteniendo a lo largo de los años, con fecha 16 de Agosto de 1949, la Comisión Municipal Permanente acuerda de conformidad con el Sr. Juez Instructor un expediente disciplinario contra las Matronas Doña María Amador Robledillo y Doña Remedios López Mesa y sancionarlas con la sanción de un mes de suspensión de empleo y sueldo durante cuyo plazo debían fijar su residencia habitual y exclusiva en las barriadas a que estaban destinadas ${ }^{15}$.

En cuanto a las diferencias de salario entre hombres y mujeres en el periodo de estudio, se encontraron diferencias de entre los Practicantes, (Profesionales sanitarios de equiparable formación a las Matronas) y las Matronas de la Beneficencia Municipal de la misma categoría (Cruz Artacho, 2009).

Tabla 3. Número de profesionales y salarios Beneficencia Municipal, año 1940.

\begin{tabular}{|c|ccc|}
\hline $\begin{array}{c}\text { Categoria cuerpo } \\
\text { sanitario de la } \\
\text { Beneficencia }\end{array}$ & $\begin{array}{c}\text { Salario Médicos. } \\
\text { Número de } \\
\text { profesionales. () }\end{array}$ & $\begin{array}{c}\text { Salario Practicantes. } \\
\text { Número de } \\
\text { profesionales. () }\end{array}$ & $\begin{array}{c}\text { Salario } \\
\text { Matronas }\end{array}$ \\
\hline Primera & (ninguno) & $7000(1)$ & (ninguna) \\
Segunda & 8000 Ptas.(4) & (ninguno) & $4000(3)$ \\
Tercera & $7000(15)$ & $5000(4)$ & $3000(2)$ \\
Cuarta & $6000(17)$ & $4000(14)$ & $2000(2)$ \\
Quinta & $5000(13)$ & $3000(22)$ & 7 \\
\hline TOTAL & 49 & 41 & \\
\hline
\end{tabular}

Fuente: AHMM (Elaboración propia)

\footnotetext{
${ }^{11}$ Archivo Histórico Municipal de Málaga. Lg 3665

${ }^{12}$ Archivo Histórico Colegio de Enfermería de Málaga. Expediente número 1 de matronas

${ }^{13,14}$ Archivo Colegio de Enfermería de Málaga. Libro I. Junta de Gobierno Colegio de Matronas

${ }^{15}$ Archivo Municipal de Málaga. Libro de Acta Capitular 1949
} 


\section{Número de Practicantes y Matronas de la Beneficencia Municipal año 1950}

\begin{tabular}{|c|ccc|}
\hline $\begin{array}{c}\text { Categoria cuerpo } \\
\text { sanitario de la } \\
\text { Beneficencia }\end{array}$ & $\begin{array}{c}\text { Salario Médicos. } \\
\text { Número de } \\
\text { profesionales. () }\end{array}$ & $\begin{array}{c}\text { Salario Practicantes. } \\
\text { Número de } \\
\text { profesionales. () }\end{array}$ & $\begin{array}{c}\text { Salario } \\
\text { Matronas }\end{array}$ \\
\hline Primera & (ninguno) & $7000(1)$ & (ninguna) \\
Segunda & 8000 Ptas.(4) & (ninguno) & (ninguna) \\
Tercera & $7000(15)$ & $5000(4)$ & $4000(3)$ \\
Cuarta & $6000(17)$ & $4000(14)$ & $3000(2)$ \\
Quinta & $5000(13)$ & $3000(22)$ & $2000(2)$ \\
\hline TOTAL & 49 & 41 & 7 \\
\hline
\end{tabular}

Fuente: AHMM (Elaboración propia)

Se observaron también diferencias salariales en las retribuciones del SEDEM (Seguro de Enfermedad de Empleados Municipales), cuyas retribuciones eran de 2.08 pesetas por cartilla y mes y para las Matronas la mitad ${ }^{16}$.

Las profesionales malagueñas de la Beneficencia Municipal era un colectivo reivindicativo. (González Castillejo, 2001), tal y como demuestran las numerosas cartas encontradas en Archivo y dirigidas a la Corporación $\mathrm{Mu}$ nicipal, pidiendo aumento de salarios, mejoras laborales y subidas de categoría profesional.

El 22 de Febrero de 1932, Doña Concepción de la Puerta Martín, Matrona Numeraria de la Beneficencia Municipal en la Casa de Socorro Santo Domingo, solicita al Ayuntamiento de Málaga le sea abonada la locomoción de servicios de larga distancia a altas horas de la noche. La Corporación Municipal acuerda estudiarlo para los presupuestos de 1933, pero finalmente el 25 de Julio de 1932, se concede servicio de carruajes para Matronas en casos urgentes que se encuentren a distancia y solo en las horas nocturnas ${ }^{17}$.

En 1924, un grupo de seis Matronas, dirigen una carta al Sr. Alcalde con una petición de aumento de sueldo, argumentándolo en función de la carestía de la vida ${ }^{18}$. Uno de los problemas a los que se tenían que enfrentar las Matronas malagueñas era el intrusismo profesional en las distintas barriadas donde ejercían parteras que eran vecinas a los que la población estaba acostumbrada, a este respecto encontramos una carta de vecinas de la barriada de Churriana que manifiestan no querer ser asistidas por la Matrona Doña Josefa Lázaro Gómez $^{19}$. A este respecto Doña Eloisa Molero Herrera, Presidenta del Colegio de Matronas de Málaga en el año 1932, publica un artículo en un periódico local de la ciudad el 6 de Agosto de 1932. El artículo se titula “ ¿Se atacará seriamente el Intrusismo?"20

Hay una carta de esta Presidenta, con fecha 14 de Octubre de 1932, en la que se refleja que en el Hospital Provincial de Málaga, (Diputación Provincial), no solo se asisten partos de Beneficencia a las pobres de necesidad sino también a mujeres que podía pagar una $\mathrm{Ma}$ trona titulada. Se solicita que se exija el padrón de pobre para ser asistida en el Hospital con fecha 27 de Octubre de $1932^{21}$.

La población malagueña fue aceptando la aparición de las Matronas de Beneficencia en la atención al parto, prueba de ello es la carta que dirige un grupo de vecinos de la Carihuela (Torremolinos), a la Corporación Municipal el

\footnotetext{
${ }^{16}$ Archivo Histórico Municipal de Málaga. Acta Capitular L-367. Página 69.

17,18,19 Archivo Histórico Municipal de Málaga. Lg 3654.

${ }^{20}$ Archivo Colegio de Enfermería de Málaga. Expediente de Eloisa Molero.

${ }^{21}$ Archivo Diputación Provincial de Málaga Lg 1028/60.
} 
27 de Mayo de 1932, solicitando se nombre a Doña Carmen Aranjuez Segura como Matrona de la Beneficencia ya que la Matrona titular de Torremolinos por estar ocupada o por la distancia no llegaba a tiempo a los nacimientos. El Ayuntamiento desestima la petición ${ }^{22}$.

Las Matronas Municipales no tenían derecho a baja por enfermedad, si enfermaban debían pedir un permiso al Ayuntamiento para ausentarse de su puesto de trabajo, a este respecto encontramos varias solicitudes ${ }^{23}$.

La jubilación era a los 75 años con lo que muchas de ellas morían ejerciendo su actividad laboral.

En caso de gestación trabajaban hasta el $8^{\circ}$ $9^{\circ}$ mes y tenían un permiso posterior al parto solo de 40 días $^{24}$.

En el área rural de la provincia de Málaga, el trabajo de las matronas de Beneficencia era una constante lucha contra las adversidades que rodeaban la asistencia sanitaria en la España de aquellos momentos. La falta de medios, los desplazamientos, la incultura de la población, las dificultades de género y la gran responsabilidad de su trabajo lejos de toda Clínica u Hospital, hacía que el trabajo diario de estas profesionales pioneras del trabajo femenino fuera extremadamente difícil (Amezcua, 2002) (Andina Díaz, 2003) (Linares Abad \& Moral Gutiérrez, 2005) (Urmeneta Marín, 2000). Además debían luchar debían contra el intrusismo que ejercían de las parteras locales, que eran mujeres que asistían los partos en los pueblos, que gozaban de la confianza de sus vecinas pero que no tenían ninguna formación académica (Linares Abad \& Moral Gutiérrez, 2005). A este respecto encontramos numerosas cartas personales en el Archivo del Colegio de Matronas de Málaga como la de Doña Isabel Cuesta Plaza que denuncia el intrusismo de la aficionada del pueblo donde trabaja y a la cual incluso favorecen los Médicos locales: “...no por llevar más de un año en el pueblo, he podido conseguir que la intrusa deje de trabajar, hace dos días hizo el parto de la hija de un médico y unos cuantos más de los mejores..." ${ }^{25}$.

Los pueblos de la provincia de Málaga se dividían en primera categoría, segunda y tercera dependiendo del número de habitantes, $y$ el salario de las matronas oscilaba entre $1200 \mathrm{y}$ 900 pesetas en el año $1934^{26}$. Los pueblos que ocuparon rápidamente, sus plazas de Matrona fueron los de primera categoría lentamente se fueron ocupando algunos de segunda, mientras que en los de tercera quedaba en su mayoría sin cubrir el puesto.

En nuestra investigación encontramos que el total de pueblos de la provincia de Málaga donde existía la figura de la matrona era en un total de 46 , mientras que el total de localidades con plaza de matrona ofertada era de 100 .

La localidad que tuvo la primera plaza de matrona de la Beneficencia Municipal en la provincia de Málaga fue Antequera, esta plaza fue ocupada por Doña Luisa Fernández Arjona García desde el 3 de enero de $1917^{27}$.

Las matronas de pueblos de segunda y tercera categoría tenían dificultades para el cobro de su salario de Matrona de la Beneficencia pagado por el Ayuntamiento, así lo demuestran cartas

\footnotetext{
${ }^{22}$ Archivo Histórico Municipal de Málaga Lg 3654.

${ }^{23}$ Archivo Histórico Municipal de Málaga. Lg 3655 y Acta Capitular L354.

${ }^{24}$ Archivo Histórico Municipal de Málaga. Acta Capitular L-354. 20 de Noviembre de 1947.

${ }^{25}$ Archivo Colegio Oficial de Enfermería de Málaga. Expediente de Matrona número 71.

${ }^{26}$ Archivo Colegio de Enfermería de Málaga. Junta de Mancomunidad De Municipios de Málaga. 28 de Noviembre de 1934.

${ }^{27}$ Archivo Municipal de Antequera. Expediente personal Doña Luisa Fernández Arjona.
} 
dirigidas al Colegio de Matronas, para ilustrar este aspecto unas palabras de Doña Francisca Borrego matrona del pueblo de Archidona entre 1940 a 1943: "Es verdaderamente lamentable que después del sacrificio que para mí representó el estudio de esta carrerita con la que tengo que sostener a mi familia y después de trabajos enormes con ellos pudiera conseguir alguna retribución, después del tiempo trascurrido no me lo hayan abonado, ni se sepa cuando ni como se me van a abonar"28.

\section{CONCLUSIONES}

La incorporación de la Matrona al escenario sanitario permitió una cobertura durante el proceso reproductivo a las mujeres pobres de Málaga a través de la Beneficencia Municipal.

Las diferencias salariales entre Matronas y Practicantes eran importantes tanto en los salarios de la Beneficencia como del Seguro de Enfermedad de Empleados Municipales.

A pesar del escaso número de Matronas en la red de Beneficencia Municipal, el colectivo no era un grupo profesional pasivo, sino reivindicativo como se demuestra en la amplia documentación de archivo encontrada: numerosas quejas dirigidas al Colegio Profesional, al Ayuntamiento y escritos a la prensa reclamando sus derechos.

El lento desmantelamiento de la asistencia domiciliaria del parto a favor de la asistencia hospitalaria fue restando autonomía al trabajo de las Matronas de la Beneficencia Municipal de Málaga.

Las Matronas del área rural de Málaga, tenían que enfrentarse a un mayor número de dificultades que las de la Capital.

\section{BIBLIOGRAFÍA}

- Amezcua, M. (2002). Memorias de una partera tradicional. Francisca Santos Olmo," Paca la Cachorra. Index de Enfermería [Index Enferm], 38(40). Recuperado de: http://www.aamatronas.org/web/htmls/noticias/noticias07/la_cachorra.pdf

- Andina Díaz, E. (2003). Los cuidados prestado por las matronas en el Bierzo Alto (León): cien años de evolución. Cultura de los cuidados: Revista de enfermería y humanidades, (13), 12-22.

- Cruz Artacho, S. (coord). (2009). La mujer trabajadora en la Andalucía Contemporánea (1931-2007). Andalucía: UGT.

- Giménez Reyna, F. (1989). Memorias de un cirujano rural: (Antequera y Malaga en la $1^{a}$ mitad del s.XX). Málaga. Ed.Universidad de Málaga (UMA).

- González Canalejo, C. (2003). Las mujeres como agentes de asistencia social. En VII Taller de la AEIHM. Almería.

- González Castillejo, M. J. (2001). Las Matronas Malagueñas un colectivo profesional discriminado en la Dictadura de Primo de Rivera (1923-1930). En Actas del III Congreso de Historia de Andalucía. Córdoba: Obra Social y Cultural Cajasur.

- Linares Abad, M., \& Moral Gutiérrez, I. (2005). Las gestoras del nacimiento en el siglo XX. el caso de Bélmez de la Moraleda. Sumuntán, (22), 31-40.

- Luque, C. C. (2000). La transición demográfica en el primer tercio del siglo XX. Cuadernos de ciencias económicas y empresariales. Papeles de trabajo, (25), 1.

- Martínez Alcubilla, M. (1886). Diccionario de la Administración Española. (Vol. Tomo I). Madrid.

- Martínez-Hernáez, À., Masana, L., \& DiGiacomo, S. M. (2013). Evidencias y narrativas en la atención sanitaria: Una perspectiva antropológica. Universitat Rovira i Virgili.

- Orengo Díaz del Castillo, F. (1953). Obstetricia para Comadronas. $2^{a}$ Edición (2a ed.). Madrid: Editorial Científico Médica Española.

- Sánchez-albornoz, S. C. (1985). Legislación protectora de la maternidad en la época de la Restauración española. Cuadernos de Historia Moderna y Contemporánea, 6, 147.

- Urmeneta Marín, A. (2000). Comadronas y cirujanos navarros en el siglo XVIII: pleito de una partera ante el intrusismo de un cirujano. Híades: Revista de Historia de la Enfermería, (7), 317-325.

- Vega, Eulalia. (2007). Mujeres y asociaciones obreras frente al seguro obligatorio de enfermedad durante la Segunda República. En Género y políticas del trabajo en la España contemporánea, 1836-1936 por Borderias, C. (pp. 253-275). Edicions Universitat Barcelona.

- Vidal Galache, F. V. (1987). El impacto de la Ley General de Beneficencia de 1822 en Madrid. Revista de la Facultad de Geografía e Historia, 1. Recuperado a partir de http://e-spacio.uned.es/fez/eserv/bibliuned:ETFSerie5424B06E2-8AAA-ACA4-8B54-FA0093105205/Documento.pdf

\footnotetext{
${ }^{28}$ Archivo Colegio de Enfermería de Málaga. Expediente de matronas número 12.
} 